\title{
Étude et réalisation de la boîte à plan de cisaillement vertical
}

T. MESSAS

C. COULET

J. GIELLY

R. AZZOUZ

L2M, IUT A Département

Génie Civil, Lyon 1 .

43, bd du 11-Novembre 1918,

69622 Villeurbanne Cedex,

France
Afin de représenter le mode réel de rupture lors d'un glissement d'un remblai, un essai de cisaillement original "à plan de cisaillement vertical » a été mis au point. Pour cela, un appareil a été conçu et construit. Les

caractéristiques mécaniques du sol utilisé, obtenues à l'aíde de cette boîte, sont comparées à celles obtenues à la boíte à plan de cisaillement horizontal. Les déchets de matières plastiques peuvent constituer des nappes de renforts discontinues pour améliorer les caractéristiques mécaniques des sols des remblais. Des essais effectués sur la nouvelle boite de cisaillement ont permis de quantifier la résistance à la traction apportée par ces renforts. Une correspondance a été établie avec les valeurs obtenues pour des nappes continues.

\section{Study and realization of a box to plan of vertical shear}

The real way of rupture during embankment failure was simulated by an original shear test : test with vertical shear plan. An apparatus was conceived and built specially for this purpose The obtained characteristics of used soils by this new test were compared with other one, carried out by the direct shear test. Waste plastic materials can be used as discontinuous reinforcement layers in order to improve the mechanical features of embankment soils. Tests were performed on a shear box machine equipped with a vertical failure plan which closely represents the real stress fields. These tests were tightiy connected with the values resulting from the study of continuous lavers.
} 


\section{Introduction}

L'étude du renforcement des sols à l'aide de l'essai à plan de cisaillement horizontal imposé ne modélise pas fidèlement la réalité d'un remblai renforcé, dans lequel la ligne de glissement se propage du haut vers le bas de celui-ci (Fig. 1). La direction de la ligne de glissement est presque perpendiculaire à l'orientation des nappes.

Devant ce problème, un essai de cisaillement original «à plan de cisaillement vertical » a été mis au point. Un appareil a été conçu et construit. Avant d'aborder les essais de cisaillement des sols renforcés à l'aide de cet appareil, nous présentons le dispositif d'essais, ainsi que son utilisation dans le cas des sols sans renforcement. Dans ces essais, la contrainte tangentielle est maitrisée, mais la difficulté rencontrée était de savoir comment mesurer la contrainte normale au plan de cisaillement. Pour pallier ce handicap, nous avons employé une technique de mesure qui consiste à utiliser un écran maintenu par quatre anneaux dynamométriques. Cet écran est confondu avec le plan de cisaillement. Lè système a été testé par plusieurs essais préliminaires.

Afin de confirmer les caractéristiques mécaniques du sol utilisé, des essais à la boîte à plan de cisaillement horizontal imposé ont été effectués. L'objectif étant de s'assurer que dans le cas des sols non renforcés, les deux types d'essais donnent des résultats comparables (principe d'objectivité).

Par ailleurs, connaissant une augmentation sensible liée à leur utilisation diversifiée et sans cesse croissante, les déchets de matières plastiques (sacs d'emballage, bouteilles...) peuvent être utilisés comme moyen de renforcement. En effet, plus de 600 millions de tonnes de déchets plastiques sont jetés chaque année en France. Dans le monde, l'accumulation de ces déchets atteindrait les 100 milliards de tonnes d'ici l'an 2050 [MAES, 1993]. Ces déchets posent de gros problèmes: leur recyclage nécessite des tris minutieux, leur élimination par incinération causant des dangers irréversibles à l'environnement, et leur mise en décharge est coûteuse. Le procédé Plasterre (Coulet et al., 1987) permet de valoriser les déchets de plastique souple en les rémployant comme structure de renfort pour améliorer les caractéristiques mécaniques des sols de remblais. Les nappes de déchets, régulièrement espacées sur la hauteur du remblai, sont constituées d'éléments discontinus dont il faut déterminer le comportement. L'approche expérimentale est abordée avec des essais comparatifs entre des nappes d'éléments discontinus et des nappes de feuilles continues. La question qui se pose avec ce procédé est de savoir comment déterminer la résistance à la traction d'une nappe constituée d'éléments discontinus de matières plastiques. Pour y répondre, nous avons étudié ce problème à l'aide d'essais de cisaillement originaux (à plan de rupture vertical). Nous examinons l'influence de plusieurs paramètres : la quantité des morceaux de plastiques par nappe, la taille des morceaux de plastiques ainsi que l'influence du mode de fixation. Le but de ces essais est de faire la liaison entre le renforcement par nappes discontinues (qui représente l'application du procédé in situ), et le renforcement par nappes continues (qui représente la partie " calcul et dimensionnement des ouvrages »). Après la présentation des résultats ainsi que de leurs analyses, une interprétation théorique du gain en cohésion sera effectuée.

\section{Présentation des matériaux et du matériel}

\section{1}

\section{Matériaux}

Le sol utilisé dans notre programme d'essais est une grave roulée provenant des carrières de la région lyonnaise. Pour obtenir une meilleure reproductibilité des essais, le sol est étuvé pour assurer une teneur en eau nulle. En effet, la teneur en eau pouvant avoir une certaine influence sensible sur la cohésion du sol ou la densité sèche il est plus simple de considérer des échantillons secs.

\section{2}

\section{Matières plastiques}

Le renforcement de la grave a été effectué par des nappes de deux types de déchets:

- feuilles de polyéthylène translucide de couleur blanche, de masse surfacique égale a $125 \mathrm{~g} / \mathrm{m}^{2}$ et d une résistance de traction à la rupture de $1,8 \mathrm{kN} / \mathrm{m}$;

- déchets de matières plastiques souples constitués essentiellement de sachets d'emballage.

\section{3}

\section{Description et caractéristiques de l'appareillage}

L'appareillage (Fig. 2) est constitué :

- de la cuve ;

- du système de cisaillement ;

- du système de chargement vertical du matériau.

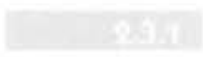

\section{La cuve}

Les dimensions de la cuve sont : $600 \mathrm{~mm}$ de longueur, $400 \mathrm{~mm}$ de largeur, $465 \mathrm{~mm}$ de profondeur. Ses parois sont très rigides afin que soient supprimées toutes les vibrations. Les parois latérales sont munies de deux fentes en leurs milieux pour permettre le passage de l'inclusion. Afin de visualiser les déplacements à l'intérieur, la paroi frontale est constituée d'une vitre qui résiste à la poussée du sol. Pour rassembler les deux zones (active et passive) du massif lors du glissement de celui-ci, cette cuve est formée par deux boites de dimensions identiques ( $300 \mathrm{~mm} \times 400 \mathrm{~mm} \times 465 \mathrm{~mm}$ ); l'une (boite $n^{\circ}$ 2) mobile et l'autre (boite $\left.n^{\circ} 1\right)$ fixe. Elles sont supportẻes par des portiques de grandes rigidités. La hauteur de ces derniers est suffisante pour installer le vérin qui permet de déplacer la boîte $n^{\circ} 2$.

\section{Tish}

\section{Le système de cisaillement}

Un vérin mécanique d'une capacité de $50 \mathrm{kN}$, ancré en position verticale permet de déplacer la boîte $n^{\circ} 2$. 


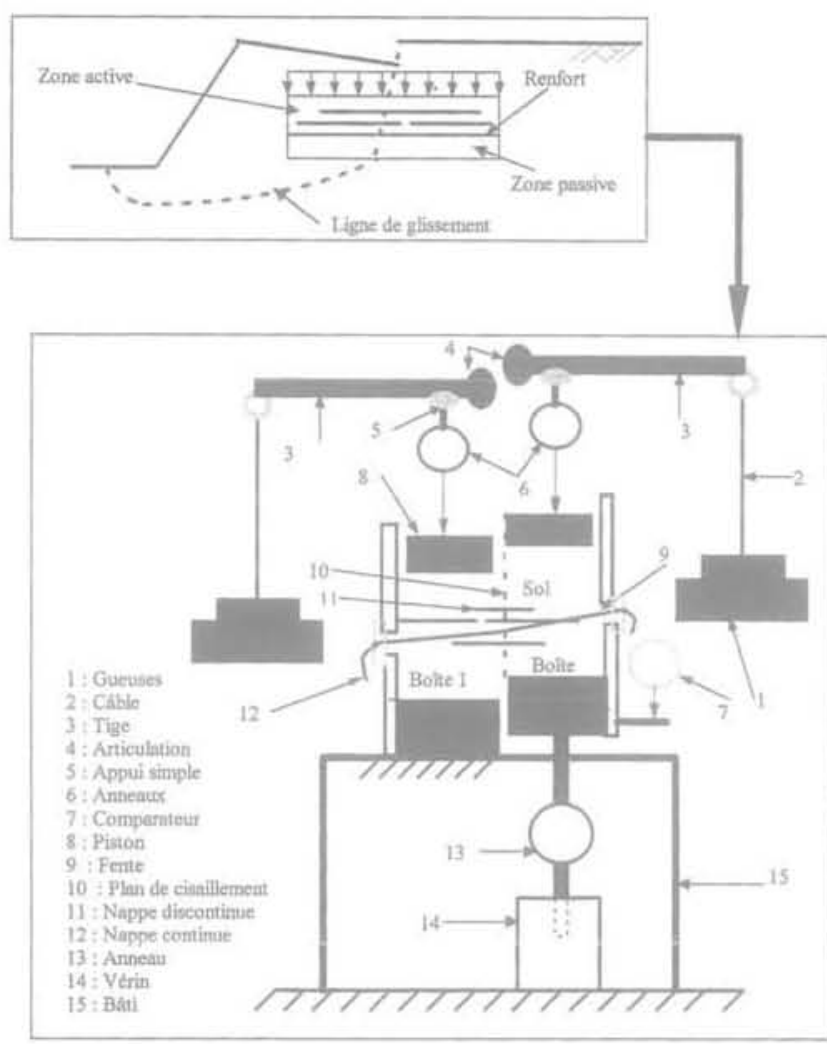

FG. 1 Glissement d'un remblai.

Embankment sliding.

HG.2 Schéma de principe du dispositif expérimental de l'essai à plan de cisaillement vertical.

Diagram of the experimental cievice of the vertical plan shearing test.

Pour l'ensemble de ces essais, la vitesse est de $1 \mathrm{~mm} / \mathrm{mn}$. Afin de mesurer l'effort de cisaillement, nous avons incorporé un anneau dynamométrique entre le vérin et la boîte $n^{\circ} 2$. Sur le bord supérieur de celle-ci, nous avons placé un comparateur de course de $100 \mathrm{~mm}$ qui permet de suivre le déplacement vertical.

\section{Le système de chargement vertical du matériau}

Le système de chargement propre est composé de gueuses supportées par un câble accroché à une tige de grande rigidité. Cette tige est posée sur l'anneau. Ce dernier affiche l'effort normal qui existe sur le plateau transmettant une charge au sol.

\section{Mise en place d'une manipulation}

Après avoir déterminé la quantité đu gravier $5 / 12$ nécessaire pour former le massif de dimensions : $600 \mathrm{x}$ $400 \times 220 \mathrm{~mm}^{3}$ et de densité 1,70; nous préparons le plastique et procédons à la mise en place comme suit : - remplissage de la cuve : la mise en place du matériau sol s'effectue par couches de $55 \mathrm{~mm}$ que l'on compacte avec une dame carrée de $80 \times 80 \mathrm{~mm}^{2}$ afin d'obtenir la densité voulue. Les nappes de plastiques sont soigneusement intercalées aux emplacements prévus ;
- mise en place du système de cisaillement et de chargement:

- mise en place des comparateurs de mesure de déplacement.

\section{3}

\section{Étude de cisaillement du sol non renforcé}

\section{Détermination de la contrainte normale au plan de cisaillement grave-grave}

Les contraintes normales au plan de cisaillement sont déterminées comme le montre la figure 3. L'écran $\mathrm{AB}$ est un panneau en duralumin, de dimensions : $465 \mathrm{~mm}$ (hauteur) $\times 396 \mathrm{~mm}$ (largeur) $\times 10 \mathrm{~mm}$ (épaisseur). Son contact avec le massif, de densité $\rho=1,7$, se fait sur une surface de $220 \mathrm{~mm} \times 396 \mathrm{~mm}$. L'écran est rendu rugueux grâce au collage des grains de gravier de même nature. L'écran est suspendu par son sommet $\mathrm{A}$ et soutient un remblai horizontal. Il peut se déplacer, mais son déplacement ne permet pas à tout le coin de glissement d'entrer en plasticité parfaite. Il est maintenu par quatre anneaux dynamométriques. L'anneau 5 permet la mesure de la force verticale (l'effort tangentiel sur l'écran), et donc par la suite celui de l'inclinaison moyenne des contraintes. Cet anneau travaille d'abord en traction lors de la mise en charge clu massif, puis en compression pendant le cisaillement. Les anneaux 2, 3, 4, travaillent en compression et sont solidaires de la contre-paroi. Ils permettent de mesurer les forces horizontales (efforts normaux sur l'écran). Un léger jeu subsiste entre l'écran et les autres parois de la cuve. L'étanchéité a été obtenue en plaçant des joints en plastique. Pendant la construction du massif, après la mise en cuvre de chaque couche suivie de son compactage, on fait une lecture des anneaux. Ceci permet de suivre l'évolution des efforts transmis par le sol à l'écran, en fonction de la hauteur du sol, au cours de la réalisation du modèle, Les valeurs de ces efforts sont faibles, étant donné la précision des anneaux et la flexibilité de l'écran qui joue dans le sens de la diminution de ces efforts. Ensuite, on procède à la pose des surcharges. On constate alors que la transmission ne se fait qu'à partin d'un certain seuil de chargement. Ceci est dû probablement à des effets parasites de contact entre le gravier et le piston.

Pour mettre l'écran en poussée, on active le vérin (Fig. 3 ) en donnant un déplacement de cisaillement de la boite $n^{\circ} 2$ jusqu'à la rupture de l'échantillon du massif. A chaque millimètre de déplacement, on fait une lecture des anneaux dynamométriques. L'ensemble des mesures permet d'avoir l'évolution des efforts sur l'écran en terme d'intensité, de direction et de point d'application en fonction des déplacements du massif. On désigne respectivement par $F_{2}, F_{3}, F_{4}$ et $F_{x}$ les efforts enregistrés par les anneaux $(2,3,4$ et 5$)$. L'effort résultant F peut être déterminé en terme de module, de direction et de position, par les expressions suivantes: - module $|\mathrm{F}|=\left[\left(\mathrm{F}_{2}+\mathrm{F}_{3}+\mathrm{F}_{4}\right)^{2}+\mathrm{F}_{5}^{2}\right]^{1 / 2}$;

- direction par rapport à la normale à l'écran $\delta=\operatorname{arctg}$

$\mathrm{F}_{5} /\left(\mathrm{F}_{2}+\mathrm{F}_{3}+\mathrm{F}_{4}\right)$; 


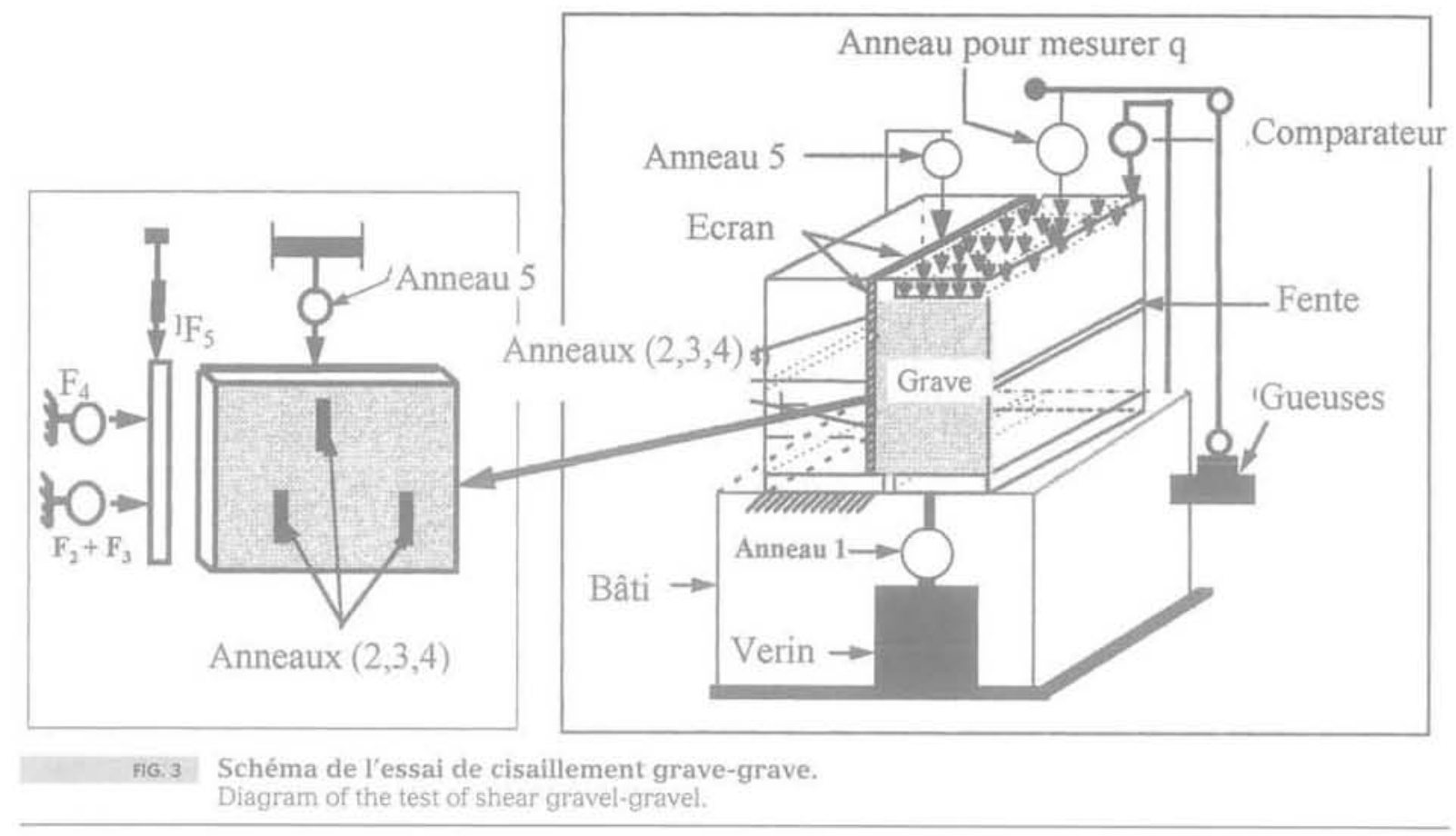

- position par rapport au pied de l'écran

$\left.F_{3}\right) \cdot Z_{(F 2+F 3)}+F_{4} \cdot Z_{F 4} /\left(F_{2}+F_{3}+F_{4}\right)$.

(B) $\mathrm{Z}_{\mathrm{F}}=\left(\mathrm{F}_{2}+\right.$

Pour notre modèle : $Z_{\mathrm{F} 4}=150 \mathrm{~mm}, Z_{\mathrm{FF} 2+\mathrm{F} 33}=70 \mathrm{~mm}$; - par ailleurs, à la profondeur $\mathrm{h}$ de l'écran la contrainte verticale est de : $\sigma_{\mathrm{y}}=\mathrm{q}_{2}+\mathrm{P}_{\mathrm{s}} /(\mathrm{L} . \mathrm{b})$

$q_{2}$ : la surcharge appliquée sur la zone active (demiboîte $\mathrm{n}^{\circ} 2$ ), $\left.\mathrm{q}_{2}=29,5 ; 51 ; 69 ; 96\right) \mathrm{kPa}$

le poids propre du sol de la zone active est de: $\mathrm{P}=$

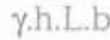

Les dimensions de l'échantillon sont: $h=0,22 \mathrm{~m}$; $b=0,4 m ; L=0,30 m$ ).

La contrainte normale à l'écran est égale au rapport de la somme des efforts normaux mesurés par les anneaux (2, 3 et 4$)$ à la surface de cisaillement (S) :

$$
\sigma=\left(\mathrm{F}_{2}+\mathrm{F}_{3}+\mathrm{F}_{4}\right) / \mathrm{S}_{\mathrm{c}}
$$

Pendant l'essai, nous avons imposé un déplacement sur l'écran $A B$. L'effort tangentiel sur l'écran est mesuré de deux manjères :

- soit par l'anneau vertical ( $\left.n^{\circ} 5\right)$;

( $\gamma$ étant le poids spécifique du gravier égal à $17 \mathrm{kN} / \mathrm{m}^{3}$ ).

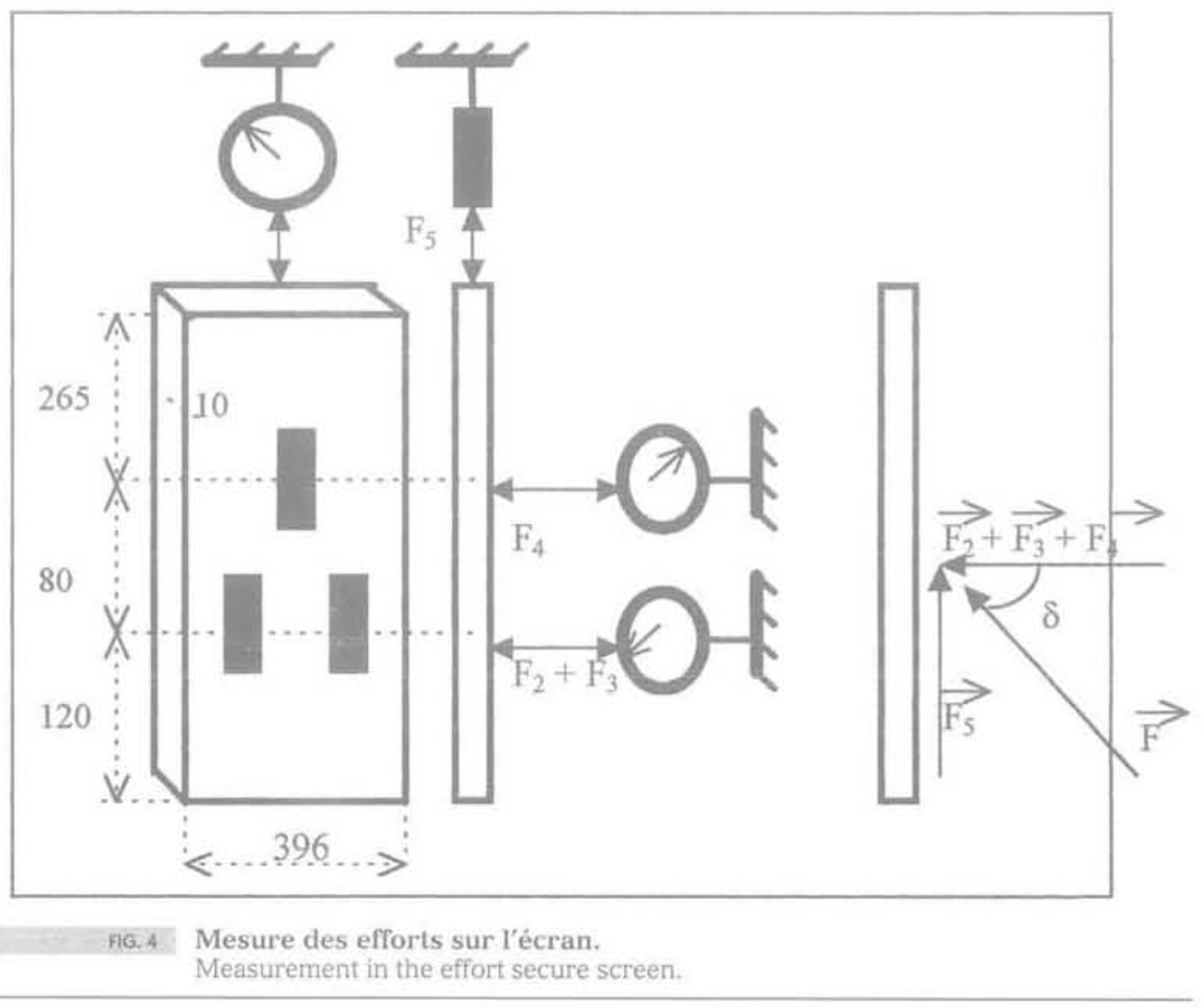


- soit par l'anneau ( $\left.n^{\circ} 1\right)$ incorporé entre le vérin et la boite mobile $n^{\circ} 2$.

Le tableau I représente, pour différents cas de chargement, les valeurs des contraintes verticales, les valeurs des contraintes horizontales, et les valeurs expérimentales des coefficients de frottement des terres au repos, ainsi que les valeurs des coefficients théoriques déduits de la formule:

$$
K_{0}=[1-2 / 3(\sin \phi)][(1-\sin \phi) /(1+\phi)]
$$

On remarque que les valeurs théoriques de $K_{0}$ sont supérieures aux valeurs expérimentales. Cette différence est due à divers facteurs :

- la flexibilité de l'écran qui joue dans le sens de la diminution de la poussée mesurée et par conséquent du $\mathrm{K}_{0}$ expérimental ;

- la sensibilité des anneaux dynamométriques;

- le frottement sur l'écran et les parois latérales, climinuant l'effort sur l'écran ;

- les incertitudes sur les mesures expérimentales.

La figure 5 représente des courbes caractéristiques de l'évolution de la contrainte normale en fonction du déplacement $(\Delta \mathrm{L})$. On constate que pour un déplacement nul, la valeur de la contrainte normale initiale sur l'écran n'est pas importante. Cela s'explique par la grande rigidité de i'écran et la faible hauteur du massif.

On remarque une augmentation notable des contraintes normales dès la mise en mouvement et ce, pour des déplacements croissants. Ceci se traduit par le phénomène de dilatance.

\section{2}

\section{Étude du frottement grave-grave}

On représente sur la figure 6, l'évolution de la contrainte de cisaillement en fonction du déplacement. On peut noter l'existence de pics très prononcés de contraintes tangentielles. Les déplacements correspondant à ces pics sont compris entre 20 et $23 \mathrm{~mm}$. La contrainte de cisaillement à la rupture augmente évidemment quand la contrainte normale initiale s'accroitt pour ensuite décroître jusqu'à atteindre la valeur résiduelle. A travers ces essais, deux remarques peuvent être formulées :

- la contrainte normale évolue en fonction des déplacements ; cela se traduit par le phénomène de dilatance.

Afin d'étudier le frottement apparent en isodéplacement, la figure 7 représente la relation $\tau / \sigma=f(\Delta L)$;

TABLENI Valeurs théoriques et expérimentales des contraintes verticales et normales, et valeurs des coefficients de frottement expérimentaux et théoriques des terres au repos.

Experimental and theoretical values of the coefficient of the earth at rest.

\begin{tabular}{|c|c|c|c|c|}
\hline $\mathrm{q}(\mathrm{kPa})$ & 29,5 & 51 & 69 & 96 \\
\hline$\sigma_{0}(\mathrm{kPa})$ & 8,6 & 12,5 & 16 & 21 \\
\hline$\sigma_{y}=q+\gamma^{*} h(\mathrm{kPa})$ & 33.3 & 54,4 & 72,7 & 99.5 \\
\hline $\mathrm{K}_{\text {bexpermental }}=\sigma, \sigma_{\mathrm{b}}$ & 0,26 & 0,23 & 0,22 & 0,21 \\
\hline $\begin{array}{l}K_{\text {ortherigie }}=[1-2 / 3(\sin \phi)] \\
I(1-\sin \phi) /(1+\sin \phi)]\end{array}$ & 0,37 & 0,37 & 0,37 & 0,37 \\
\hline
\end{tabular}

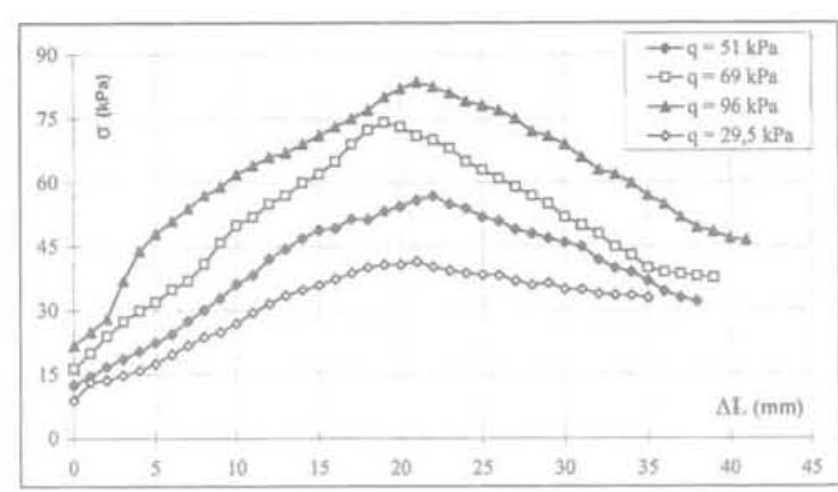

FIG.5 Evolution de la contrainte normale en fonction du déplacement.

Evolution of the normal stress versus the displacement.

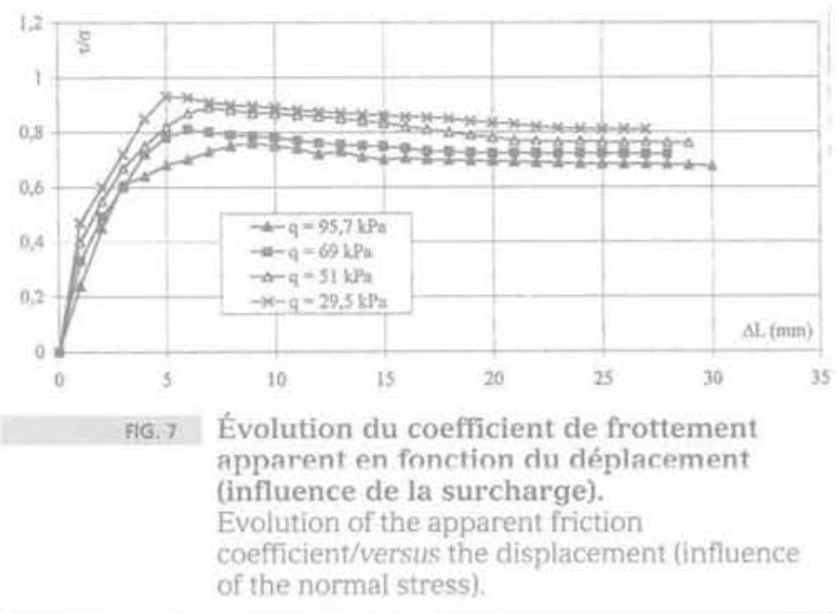

- quand la contrainte normale augmente, le coefficient de frottement apparent diminue. Ce phénomène a été observé par divers chercheurs dans le cas de plan de cisaillement horizontal. 


\section{Étude comparative des deux types d'essai de cisaillement (horizontal et vertical)}

\author{
4.1
}

\section{Appareillage d'essai \\ à plan de cisaillement horizontal}

Un appareil (une boite) permettant d'effectuer des essais bien adaptés à notre étude (Fig. 8), a été conçu. Il est composé de deux parties de largeurs et hauteurs identiques $250 \mathrm{~mm}$ et $200 \mathrm{~mm}$ respectivement. La partle inférieure, d'une longueur de 800 mm, est encastrée au sol. La partie supérieure, d'une longueur de $600 \mathrm{~mm}$, est longitudinalement mobile ; ceci afin de conserver la même surface de cisaillement (de frottement) au cours de l'essai. Il permet de tester ainsi des sols à grosse granulométrie [(D $<L / 6=100 \mathrm{~mm})$, Collios (1981)] ou d'après le critère de Kerisel (1972) L > 20D max $^{\prime} \mathrm{L}=$ cote de la boîte, et $D_{\text {max }}=$ diametre maximal des grains. Cette boite est donc bien plus grande que les boites de Casagrande classiques. De ce fait, l'influence des bords de la boitte est diminuée. On peut expérimenter le matériau sol + renforcement tel qu'utilisé in situ. La demiboîte supérieure est animée d'une vitesse constante et égale à $1 \mathrm{~mm} / \mathrm{mn}$, et ceci pour tous les essais. La traction est exercée par un vérin mécanique d'une capacité de $50 \mathrm{kN}$. A l'avant, est placé le comparateur (de course $100 \mathrm{~mm}$ ) qui permet de suivre le déplacement longitudinal. Un dynamomètre intercalé entre le vérin et la boite permet d'effectuer la mesure des efforts de cisaillement. Le chargement vertical est réalisé au moyen de gueuses de poids différents $(103,210 \mathrm{~kg} . .$. posées sur un plateau muni d'un piston de section rectangulaire afin de transmettre la charge verticale dont la valeur est fonction du nombre de gueuses posées.

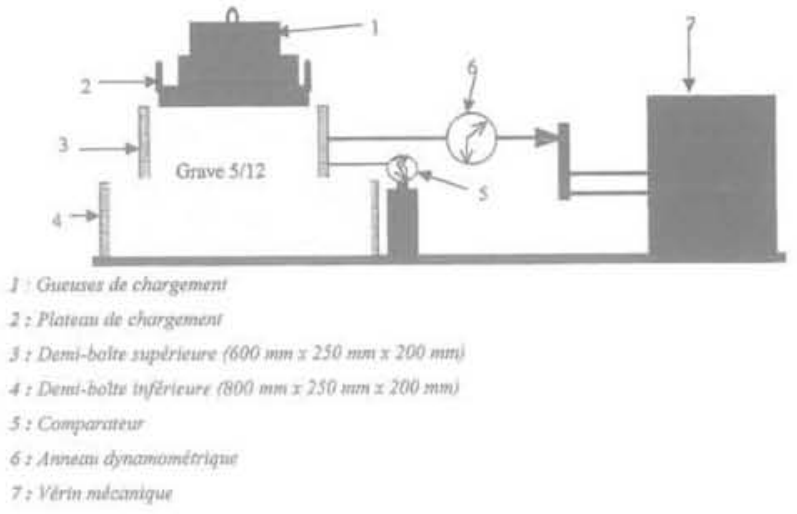

मG, B Schéma descriptif de l'appareil grand modèle de cisaillement.

Diagram of the experimental device of the horizontal plan shearing test.

\section{2}

\section{Avantages et inconvénients des deux types d'essais de cisaillement}

La boîte à plan de cisaillement horizontal est également appeiée boite de cisailiement direct du fait de sa facilité de préparation et de fonctionnement. Cet essai reste J'essai classique le plus simple à utiliser. Toutes ces facilités permettent d'étudier et de contrôler les différents facteurs. Cependant, la boite à plan de cisaillement vertical est préféré, du fait de sa représentation de la réalité lors d'un glissement de terrain. Le tableau I indique les avantages et les inconvénients de chaque type d'essai.

Dans le cas de cisaillement du sol renforcé, l'essai à plan de cisaillement vertical est préféré, vu la facilité de mise en ceuvre des nappes discontinues avec une certaine surface de recouvrement entre les éléments voisins, et l'assurance de bon ancrage des nappes continues.

TABrEAU II Avantages et inconvénients de chaque type d'essai de cisaillement (sol seul),

Adivantages and disadvantages of each type shearing test.

\begin{tabular}{|c|c|c|}
\hline Type de plan de cisaillement & Avantages & Inconvénients \\
\hline \multirow[t]{4}{*}{ Horizontal } & Procédure d'essai simple & "Ne modélise pas la rupture des sols renforcés \\
\hline & " Surface de cisaillement constante & - Concentration de contraintes aux bords \\
\hline & - Prêparation simple & $\begin{array}{l}\text { Difficulté d'étudier le cisaillement des sols ren- } \\
\text { forcés par nappes continues ou discontinues }\end{array}$ \\
\hline & - Contrainte normale $(\sigma)$ constante & \\
\hline \multirow[t]{5}{*}{ Vertical } & $\begin{array}{l}\text { Modélise mieux la rupture } \\
\text { des sols renforcès }\end{array}$ & - Systême et procédure d'essai complexe \\
\hline & $\begin{array}{l}\text { Assurance du recouvrement } \\
\text { entre nappes discontinues }\end{array}$ & -Préparation d'essai lourde \\
\hline & Bon ancrage des nappes continues & *Surface de cisaillement variable \\
\hline & $\begin{array}{l}\text { - Contróle les conditions } \\
\text { aux extrémités de la nappe }\end{array}$ & - Concentration de contraintes aux bords \\
\hline & & - Contrainte normale ( $\sigma)$ variable \\
\hline
\end{tabular}




\section{Courbes contrainte de cisaillement-déplacement}

Les résultats obtenus peuvent se distinguer les uns des autres par l'allure des courbes expérimentales (Fig. 9). La présence de pics, en terme de contrainte de cisaillement, est à noter à $\Delta \mathrm{L}$ compris entre 16 et $25 \mathrm{~mm}$ dans le cas de cisaillement à plan de rupture horizontal. et entre 20 et $23 \mathrm{~mm}$ dans le cas d'un essai à plan de cisaillement vertical. Dans celui-ci, on empêche toute variation de volume au cours du cisaillement de l'échantillon ce qui a pour conséquence de produire des contraintes de cisaillement très élevées. Ceci est dû au fait que le gravier au voisinage du plan de cisaillement a tendance à se déliter mais s'en trouve empêché par le gravier constituant les deux blocs. Par conséquent, la contrainte normale augmente beaucoup et permet l'existence de très grandes contraintes de cisaillement, Au début, pour l'essai à plan de rupture horizontal, le gonflement de l'échantillon provoque un soulèvement de la boite supérieure, puis d'un écoulement de grains de faibles diamètres. La contrainte de cisaillement à la rupture augmente quand la contrainte normale initiale s'accroît. Il est à noter que la contrainte de cisaillement décroît par la suite jusqu'à atteindre la valeur résiduelle.

\section{4}

\section{Analyse en isodéplacement}

Afin de suivre l'évolution des caractéristiques mécaniques tout au long de chaque déplacement, une analyse par les courbes intrinsèques d'isodéplacement a été effectuée de la même manière que l'analyse en isodéformation selon la définition donnée par Long (1972). Les onze valeurs suivantes du déplacement $\Delta \mathrm{L}$ ont été prises : $1,3,5,8,12,15,20,24,27,30$ et $35 \mathrm{~mm}$.

Les figures 10 et 11, représentent les droites des courbes intrinsèques qui correspondent à chaque dêplacement donné pour les essais à plan de cisaillement vertical et horizontal. L'axe des abscisses peut ètre considéré comme la courbe intrinsèque d'isodéplacement correspondant à un déplacement nul. Dans le plan $(\sigma, \tau)$, on constate que la pente des droites augmente graduellement avec le déplacement $\Delta \mathrm{L}$. Dès que le déplacement atteint environ $8 \mathrm{~mm}$, la pente de ces droites ne varie pratiquement plus. L'intersection des droites d'isodéplacements coupe l'axe des contraintes tangentielles en un point d'ordonnée 2,5 kPa, ce qui montre que la cohésion naturelle de notre matériau est négligeable. Les pentes de ces droites représentent l'angle de frottement interne $\phi$. Le tableau III donne les valeurs de l'angle de frottement interne correspondant au déplacement.

La figure 12 montre l'évolution de l'angle de frottement interne en fonction du déplacement. Ces courbes représentent trois zones de déplacement:

- Ja première zone : pour $\Delta \mathrm{L}$ allant de 0 à $10 \mathrm{~mm}$, l'angle de frottement interne augnente sensiblement. A $5 \mathrm{~mm}$ de déplacement, il atteint environ $90 \%$ de sa valeur maximale;

- la deuxième zone : pour $\Delta \mathrm{L}$ allant de 10 à $15 \mathrm{~mm}$, l'angle de frottement interne $\phi$ augmente lentement: - la troisième zone : pour $\Delta \mathrm{L}$ supérieur à $15 \mathrm{~mm}$, l'angle de frottement $\phi$ se stabilise avec quelquefois une diminution de celui-ci.

Cette approche en isodéplacement, permet d'obser-

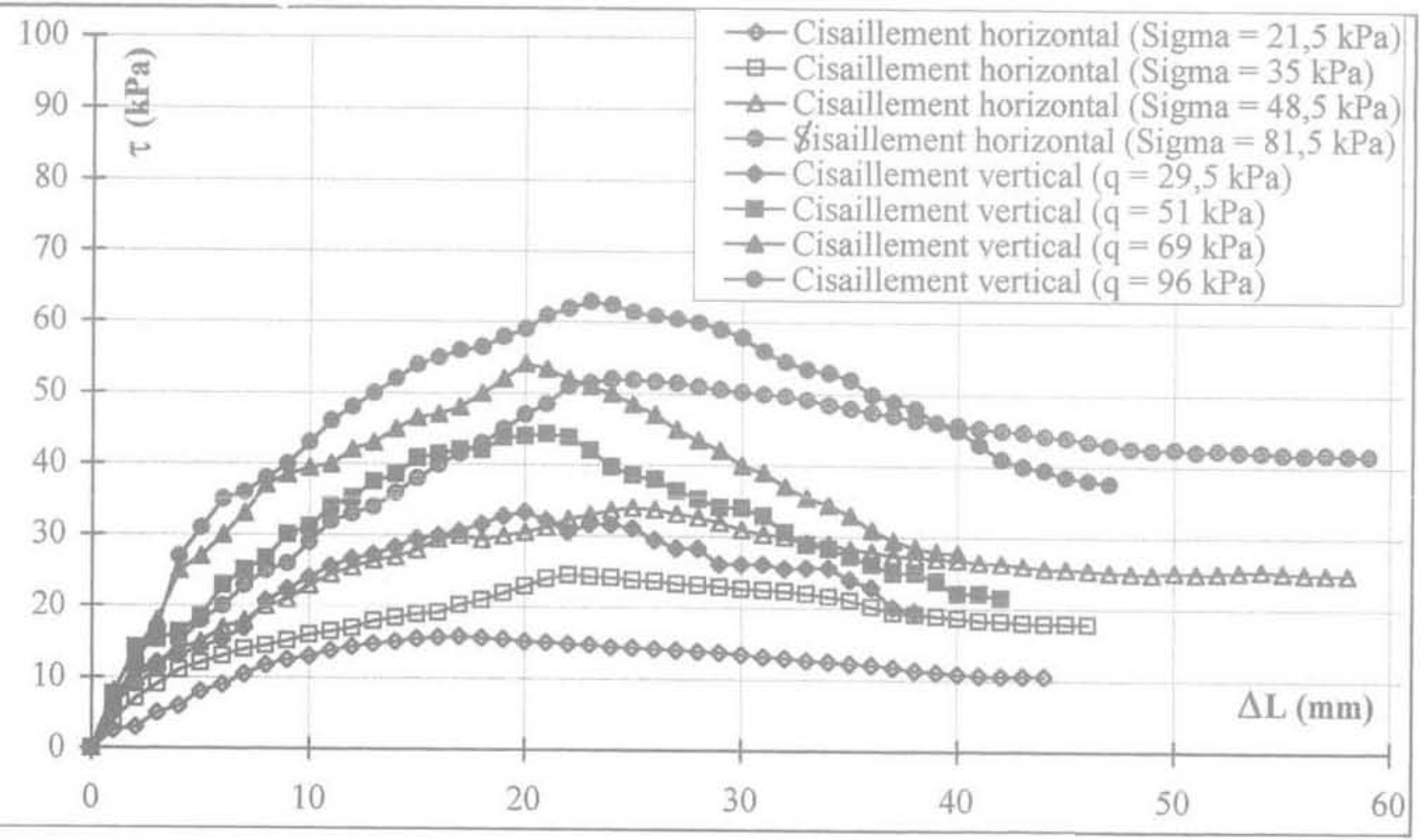

fIG. 9 Courbes contraintes de cisaillement-déplacements (essai de cisaillement grave-grave). Curves shear stress-displacement (test of shear gravel-gravel). 


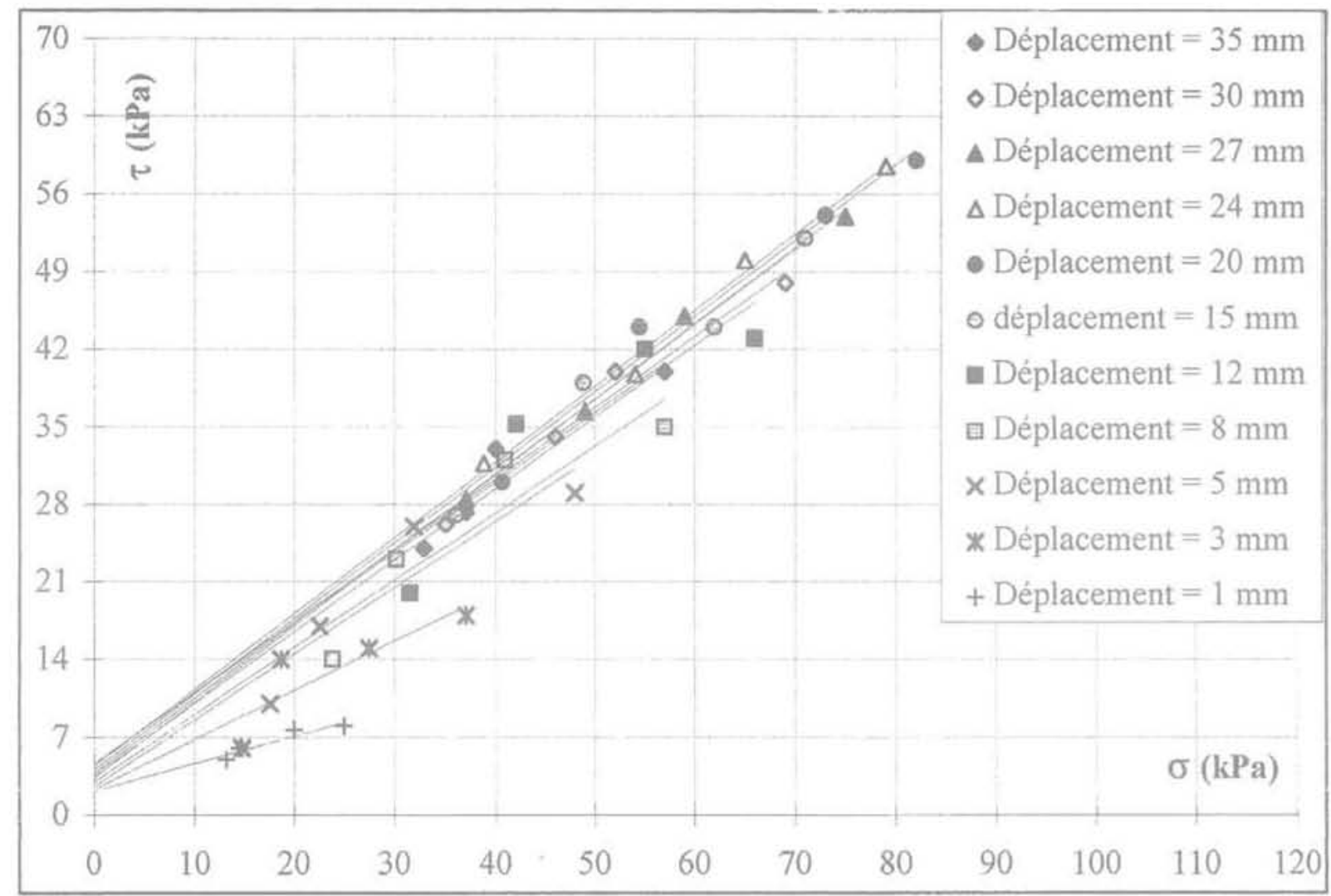

FiG,10 Droites des courbes intrinsèques d'isodéplacement (essai à plan de cisaillement vertical). Intrinsic curves of the gravel (test of the vertical plan shearing test).

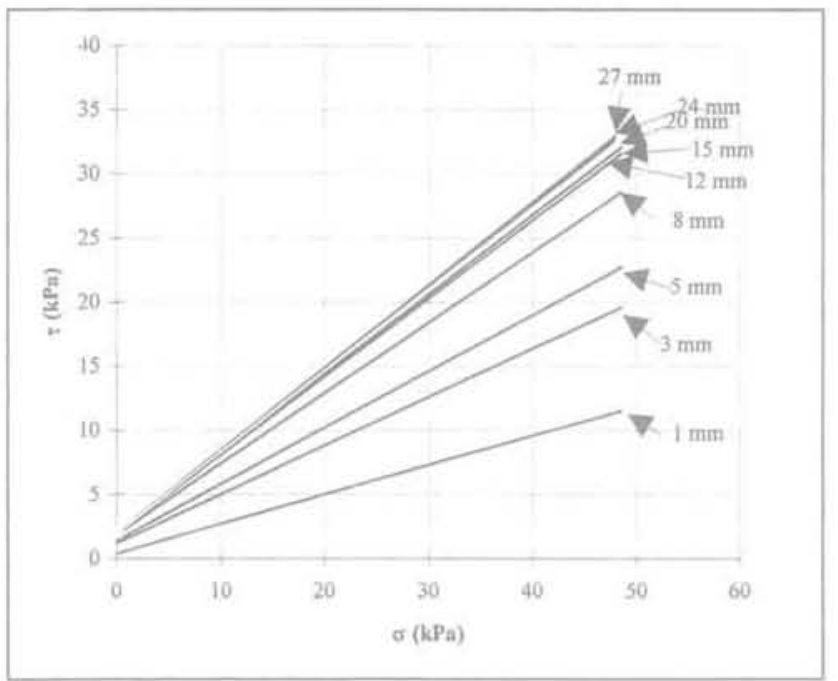

FIG,11 Droites des courbes intrinsèques d'isodéplacement (essai à plan de cisaillement horizontal). Intrinsic curves of the gravel (test of the horizontal plan shearing test). ver une augmentation rapide de $\phi$ pour les déplacements faibles ( $\Delta \mathrm{L}=0$ à $10 \mathrm{~mm})$. Ce qui correspond à la phase où la grave commence à être sollicitée mais reste dans un état élastique.

\section{5}

\section{Analyse des résultats à la rupture}

On suppose que le comportement du matériau à la rupture suit le critère de Mohr-Coulomb. Les valeurs moyennes obtenues à partir de ces essais sont résumées dans le tableau IV. A partir de la notion d'angle de frottement (Fig. 13), on constate que la valeur de l'angle de frottement moyen grave-grave, déterminée par l'essai à plan de cisaillement horizontal, est du même ordre que la valeur de l'angle de frottement interne déterminée dans un essai à plan de cisaillement vertical. L'écart entre les deux valeurs ne dépasse pas $5 \%$. Cet écart se traduit par la différence entre le mode cie rupture en cisaillement, l'état de contrainte, el les incertitudes de mesure.

TABLEAUIII Valeurs de l'angle de frottement interne $\phi$ pour différentes valeurs du déplacement $\Delta \mathrm{L}$ Values of the internal rubbing angle in function of the displacement. 


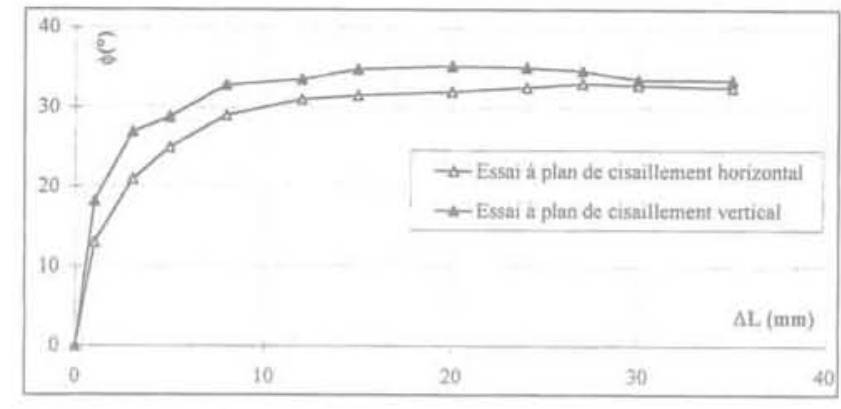

FG. 12 Mobilisation de l'angle de frottement interne $\phi$ en fonction du déplacement. Mobilisation of the internal rubbing angle $\phi$ in function of the displacement.

TABLEAUIV Caractéristiques mécaniques de la grave $5 / 12$.

Characteristic mechanical of the gravel 5,12.

\begin{tabular}{l|c|c}
$\begin{array}{c}\text { Type de plan } \\
\text { de cisaillement }\end{array}$ & $\begin{array}{c}\text { Cohésion C } \\
(\mathrm{kPa})\end{array}$ & $\begin{array}{c}\text { Angle de frottement } \\
\left.\text { interne } \phi{ }^{\circ}\right)\end{array}$ \\
\hline Horizontal & 2,3 & 33 \\
\hline Vertical & 2,5 & 35 \\
\hline
\end{tabular}

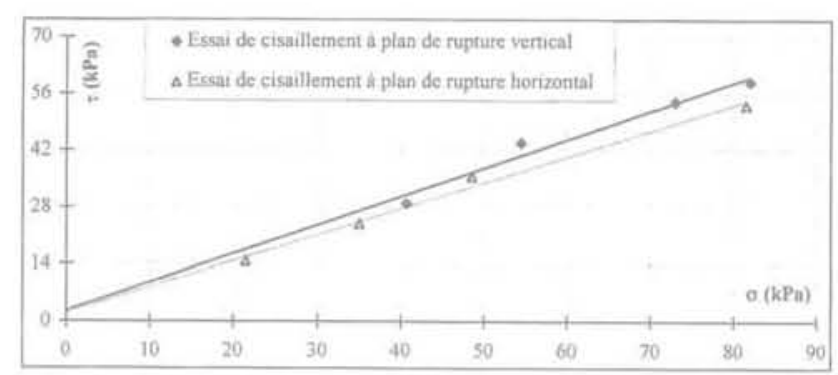

FIG.13 Courbes intrinsèques. Intrinsic curve rigths.

\section{Étude de cisaillement du sol renforcé par nappes en matières plastiques}

\section{1}

\section{Renforcement par des nappes continues}

\subsection{1.}

\section{Expérimentation : influence du mode de fixation des nappes plastiques}

Des essais de cisaillement du sol renforcé par nappes plastiques ancrées et libres ont été effectués afin de montrer l'influence de fixation des nappes.

Le tableau $V$ représente le programme de ces essais réalisés pour étudier l'influence de ce paramètre.

Les courbes contraintes-déplacements obtenues (Figs 14 et 15) présentent un comportement de type

TABLEaUy Différents types d'essais réalisés. (influence du mode de fixation des nappes plastiques).

The different types of tests made.

\begin{tabular}{c|l}
\hline No des essais & \multicolumn{1}{|c}{ Types d'essais } \\
\hline 1 & Grave seule \\
\hline 2 & $\begin{array}{l}\text { Grave renforcée par une nappe de deux } \\
\text { feuilles plastiques }\left(60 \times 40 \mathrm{~cm}^{2}\right) \text { libres }\end{array}$ \\
\hline 3 & $\begin{array}{l}\text { Grave renforcée par une nappe de deux } \\
\text { feulles }\left(60 \times 40 \mathrm{~cm}^{2}\right) \text { ancrées }\end{array}$ \\
\hline 4 & $\begin{array}{l}\text { Grave renforcée par une nappe de quatre } \\
\text { feuilles }\left(60 \times 40 \mathrm{~cm}^{2}\right) \text { libres }\end{array}$ \\
\hline 5 & $\begin{array}{l}\text { Grave renforcée par une nappe de quatre } \\
\text { feuilles }\left(60 \times 40 \mathrm{~cm}^{2}\right) \text { ancrees }\end{array}$ \\
\hline
\end{tabular}

pic-palier d'effort. Ces courbes prennent la même allure au début et divergent après un certain déplacement. Elles peuvent présenter trois phases de déplacement :

- La phase d'élasticité : c'est la phase de mise en place des grains de gravier. Au départ, pour les faibles déplacements, les courbes présentent une partie quasi linéaire. On constate que la résistance de cisaillement est la même avec ou sans nappes plastiques.

- La phase d'écrouissage : elle précède la phase de rupture. Dans cette phase, l'influence de la nappe plastique est remarquable. La résistance de cisaillement maximale du sol renforcé par la nappe ancrée est supérieure à celle obtenue par la nappe libre et qui et supérieure à la résistance du sol sans renforcement. Cette résistance varie selon le nombre de feuilles qui forment chaque nappe. Les pics des contraintes de cisaillement sont atteints pour des déplacements ( $\Delta \mathrm{l}$ ) compris entre $20 \mathrm{~mm}$ et $27 \mathrm{~mm}$. Le tableau VI indique les valeurs des contraintes de cisaillement maximales et les déplacements correspondants.

- La phase de plasticité : dans cette phase, la chute des contraintes dans le cas de cisaillement du gravier renforcé par nappes ancrées. Ceci peut s'expliquer par le fait que les feuilles plastiques ont été déchirées ; c'est la rupture et l'on se rapproche du sol non renforcé. Lorsque le pic est bien marqué, le radoucissement peut être très rapide et l'effort résiduel est alors obtenu pour des déplacements n'excédant pas $30 \mathrm{~mm}$. Dans d'autres cas, le radoucissement est très progressif et l'effort résiduel est alors atteint pour des déplacements supérieurs à $40 \mathrm{~mm}$. La comparaison de ces courbes montre que l'écart entre l'effort au pic et l'effort résiduel est d'autant plus fort que l'effort au pic est important.

Au cours de ces essais, on peut constater que les déformations des nappes ancrées sont plus importantes que celles des nappes libres. En effet, dans le premier cas, les feuilles plastiques sont nettement sollicitées par les efforts de traction provoquant les déformations. Par contre, dans le deuxième cas, la résistance de cisaillement à ces efforts est évidemment moins grande, d'où des déformations faibles et un risque de glissement des feuilles. L'influence de la surcharge se traduit par une augmentation des déplacements au pic $\left(\Delta \mathrm{l}_{\max }\right): \Delta \mathrm{l}_{\max }$ varie entre 20 et $23 \mathrm{~mm}$ pour $\mathrm{q}=51 \mathrm{kPa}$, entre 22 et $25 \mathrm{~mm}$ pour $\mathrm{q}=69 \mathrm{kPa}$, et entre 23 et $27 \mathrm{~mm}$ pour $\mathrm{q}=96 \mathrm{kPa}$. 

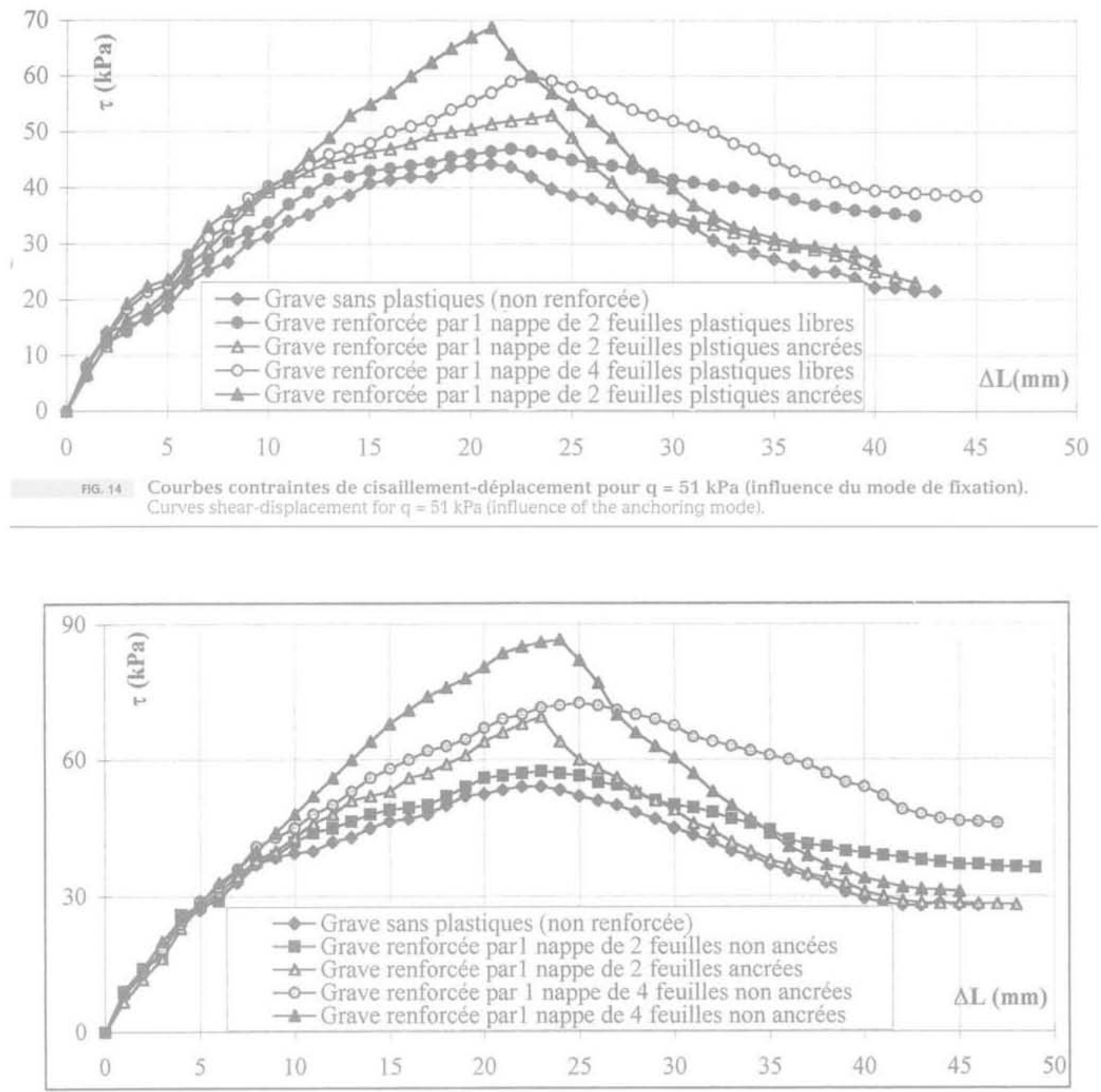

FG. 15 Courbes contraintes de cisaillement-déplacement pour $\mathrm{q}=69 \mathrm{kPa}$ (influence du mode de fixation). Curves shear stress-displacement for $\mathrm{q}=69 \mathrm{kPa}$ (influence of the anchoring mode).

TAaleauvi Valeurs des contraintes de cisaillement, des coefficients de frottement apparent $(\mu)$ et des déplacements à la rupture (influence du mode de fixation).

Vahes of shear stress, apparent coefficient friction and displacement (influence of the anchoring mode).

\begin{tabular}{|c|c|c|c|c|c|c|c|c|c|c|c|c|}
\hline \multirow[t]{2}{*}{ Type d'essal } & \multicolumn{4}{|c|}{$\mathrm{q}=51(\mathrm{kPa})$} & \multicolumn{4}{|c|}{$q=69(\mathrm{kPa})$} & \multicolumn{4}{|c|}{$\mathrm{q}=96(\mathrm{kPa})$} \\
\hline & $(\mathrm{kPa})$ & $\mu$ & $\begin{array}{c}\Delta ! \\
(\mathrm{mm})\end{array}$ & $\begin{array}{l}\mathrm{A} / \mathrm{I} \\
(\%)\end{array}$ & $\begin{array}{l}t^{t} \\
(\mathrm{kPaj})\end{array}$ & $\mu$ & $\frac{\Delta !}{(\mathrm{mm})}$ & $\begin{array}{l}\Delta / / 1 \\
(\%)\end{array}$ & $\begin{array}{c}\tau \\
\text { (kPag }\end{array}$ & $\mu$ & $\underset{(\mathrm{mm})}{\Delta !}$ & $\begin{array}{l}\Delta \mathrm{U} / \mathrm{I} \\
(\%)\end{array}$ \\
\hline 1 & 44 & 0,80 & 20 & 9 & 54 & 0,74 & 22 & 10 & 59 & 0,71 & 23 & 10.4 \\
\hline 2 & 46.7 & 0,85 & 22 & 10 & 57.8 & 0,81 & 24 & 10,9 & 63 & 0,77 & 28 & 12,7 \\
\hline 3 & 53 & 0,97 & 21 & 9,5 & 69.5 & 0,93 & 23 & 10,4 & 73,9 & 0.90 & 26 & 11.8 \\
\hline 4 & 59,9 & 1.1 & 23 & 10,4 & 72,5 & 0,99 & 25 & 11,3 & 79,6 & 0,97 & 27 & 12,2 \\
\hline 5 & 68,7 & 1,26 & 21 & 9,5 & 86,5 & 1,18 & 24 & 10,9 & 90,5 & 1,10 & 25 & 11,3 \\
\hline
\end{tabular}


Les courbes intrinsèques sont tracées dans la figure 16 à partir de la détermination des contraintes maximales de cisaillement à la rupture. On remarque que la présence des matières plastiques améliore nettement la résistance de cisaillement du sol. Les essais effectués avec des nappes plastiques non ancrées offrent une résistance moins grande que ceux effectués avec des nappes plastiques ancrées, surtout pour un déplacement important. Cette différence est la conséquence d'une efficacité considérable de la résistance à la traction des feuilles plastiques lorsque celles-ci sont ancrées.

A partir de la présentation des courbes intrinsèques et à l'aide de l'utilisation de la régression linéaire pour ces courbes, une relation entre la contrainte de cisaillement $(\tau)$ et la contrainte normale $(\sigma)$ est obtenue sous la forme suivante :

$$
\tau=a . \sigma+b
$$

a étant la pente de la droite qui représente la courbe intrinsèque avec l'axe $\sigma$, et b étant l'ordonnée de l'intersection de cette droite avec l'axe $\tau$.

On suppose que le comportement du matériau suit la loi de Coulomb : $\tau=\sigma \cdot \operatorname{tg} \cdot \phi_{\mathrm{a}}+\mathrm{C}$

En identifiant la relation (8) et (9), nous obtenons les valeurs de la cohésion $C_{\text {. }}$ (tableau VII).

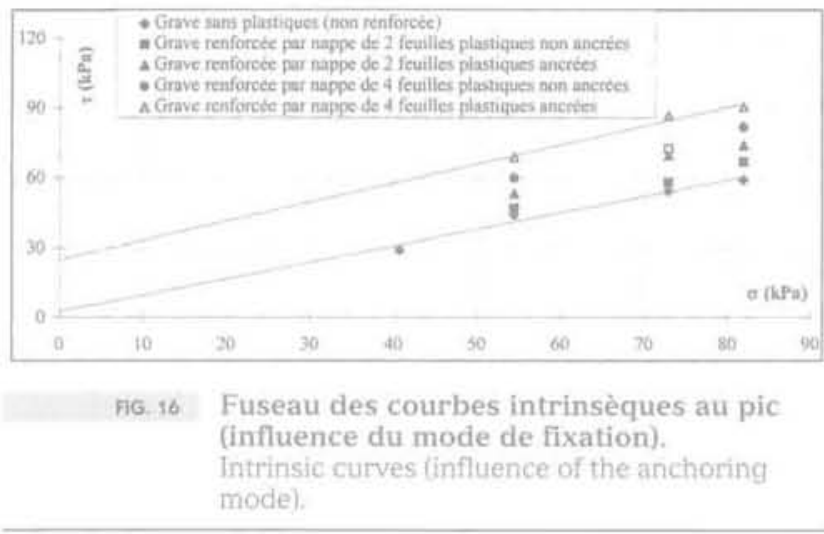

TABLEAU VII Valeurs de la cohésion (influence du mode de fixation).

Experimental values of the cohesion gain due to continuous layers.

\begin{tabular}{l|l|l|l|l|l} 
Types d'essai & 1 & 2 & 3 & 4 & 5 \\
\hline $\begin{array}{l}\text { La cohésion apparente } \\
\text { expérimentale } \mathrm{C}_{\text {ex }}(\mathrm{kPa})\end{array}$ & 2,5 & 8 & 11 & 17 & 25 \\
\hline
\end{tabular}

Le frottement apparent $\mu^{*}$ en fonction de la contrainte normale à la rupture est représenté dans la figure 17 . On constate globalement que le frottement apparent diminue quand la contrainte normale maximale $\left(\sigma_{\max }\right)$ augmente. Ce constat est en accord avec de nombreuses observations qui ont pu ètre faites dans le cadre des études sur le renforcement des sols. D'autre part, ce coefficient évolue peu avec les conditions d'essais.

\section{Calcul du gain de cohésion}

Le déplacement de la demi-boìte $n^{2} 2$ dẻveloppe deux zones d'action dans le massif : l'une est active (le sol de la demi-boite $n^{\circ}$ 2) et l'autre est passive (le sol de la demi-boite $n^{\circ}$ 1) (Fic. 18a). Nous supposons que le sol est en équilibre plastique le long du plan de rupture $A D$. Les différentes forces s'exerçant sur le prisme $A B C D$ (la demi-boite $n^{\circ}$ 2) (Fig. 18b) sont:

$\mathrm{q}_{2}$ : la surcharge appliquée sur la demi-boite $n^{\circ} 2$ (la zone active),

$\mathrm{P}_{\mathrm{s}}$ : le poids propre du sol de la zone active,

$R$ : la réaction du sol sur le plan de rupture AD. D'après l'hypothèse d'état de rupture du sol, cette réaction est inclinée d'un angle $\phi$ par rapport à la normale à plan de rupture,

$T_{v}$ : l'effort de cisaillement donné par le vérin,

$T_{v o}$ : l'effort de frottement du système à vide,

$T$ : l'effort de cisaillement effectif,

$T_{\mathrm{p}}$ : la force maximale mobilisée dans la nappe plastique,

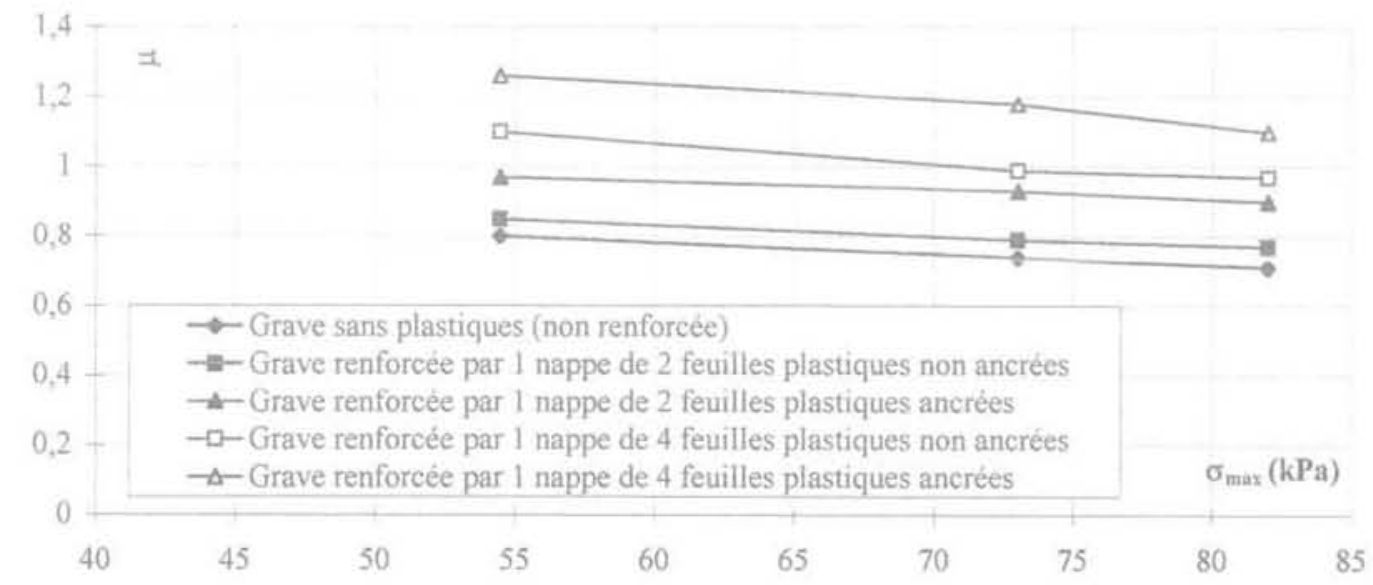

FiG.17 Variation du coefficient de frottement apparent en fonction de la contrainte normale maximale (influence du mode de fixation).

Variations of the apparent friction coefficient according to the normal tensile strength (influence of the anchoring mode), 


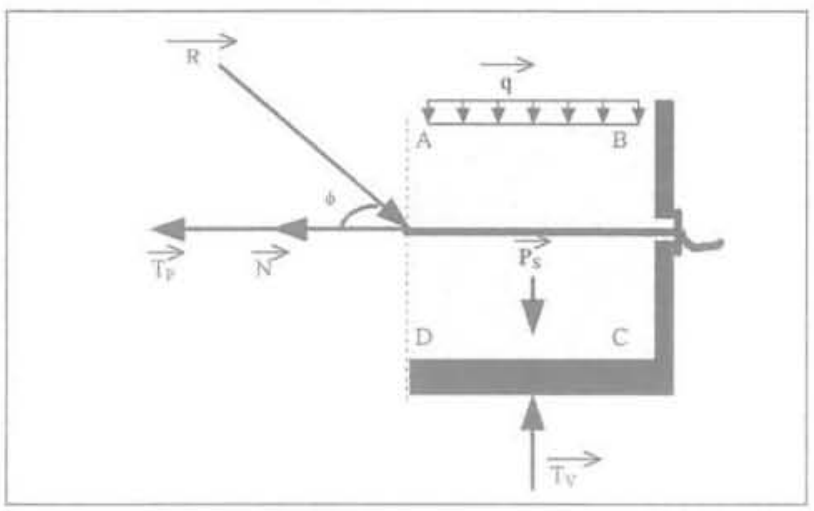

FIG. 18a Bilan des forces extérieures agissant sur le sol contenu dans la demi-boite mobile.

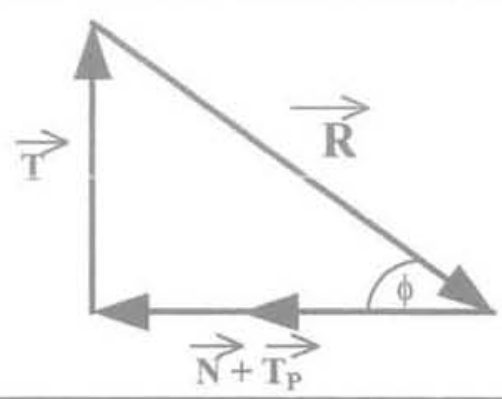

FG. 186 Diagramme d'équilibre des forces.

$\mathrm{N}$ : l'effort normal dû aux charges verticales (surcharges, poids du sol).

L'équilibre de ces forces à la rupture représenté par le diagramme de la figure $18 \mathrm{~b}$ permet d'écrire :

$$
\mathrm{T}=\left(\mathrm{N}+\mathrm{T}_{\mathrm{p}}\right) \mathrm{tg} \phi
$$

La surface de cisaillement $S_{c}$ varie en fonction du déplacement:

$$
T / S_{c}=\left(N / S_{c}+T_{p} / S_{c}\right) \operatorname{tg} \phi \rightarrow \tau=\sigma \operatorname{tg} \phi+\left(T_{p} / S_{c}\right) \operatorname{tg} \phi
$$

En identifiant la relation (11) avec la loi de Coulomb $\tau=\sigma \operatorname{tg} \phi+C$

Nous pouvons déduire l'expression de la cohésion $\mathrm{C}=\left(\mathrm{T}_{\mathrm{p}} / \mathrm{S}\right) \operatorname{tg} \phi$

Si la nappe est constituée de n feuilles de matières plastiques de largeur b ayant une résistance à la traction à la rupture $T_{p r}$, alors on pourra écrire :

$$
\Delta \mathrm{C}=\mathrm{n} \cdot \mathrm{T}_{\mathrm{pr}} \mathrm{b} \cdot \operatorname{tg} \phi / \mathrm{S}_{\mathrm{c}}
$$

$\mathrm{n}$ : le nombre de feuilles plastiques,

$\mathrm{T}_{\mathrm{pr}}$ : la résistance de la traction de feuille plastique à la rupture,

L: la largeur des feuilles plastiques,

$\mathrm{S}_{\mathrm{c}}$ : la surface de cisaillement,

$\phi$ : l'angle de frottement interne du sol.

La cohésion anisotrope du matériau composite (sol et plastique) dépend à la fois des caractéristiques à la rupture des nappes plastiques (par exemple la résistance à la traction $\mathrm{T}_{\text {J }}$ ) et de la surface de cisaillement ainsi que de l'angle de frottement interne $\phi$ de la grave utilisée.

\section{4 ins}

\section{Comparaison des résultats analytiques et expérimentaux}

Le tableau VIII indique les valeurs de la pseudocohésion $(\Delta C)$ théoriques et expérimentales apportées par la présence des matières plastiques.

Pour les essais $n^{\circ} 2$ et $n^{\circ} 4$, les valeurs théoriques du gain en cohésion ne concordent pas avec les valeurs expérimentales (les premières sont plus grandes que les secondes). Cette différence provient du phénomène suivant : l'analyse théorique est fondée sur l'état des nappes à la rupture alors que dans ces essais, la rupture des nappes n'a jamais été atteinte. Donc au lieu de prendre dans la formule $\mathrm{n}^{\circ} 14$ la résistance des plastiques en traction à la rupture $\left(T_{p}\right)$, on prend la résistance réelle $I_{p}$ à la traction au moment de la rupture de l'échantillon. 'Par contre, les deux cohésions pour les essais $n^{\circ} 3$ et $n^{\circ} 5$ concordent puisque la rupture des nappes a été atteinte.

En inversant le problème, on peut déterminer la résistance réelle à la traction $T_{p}$ des nappes de plastique en service en fonction de leur apport en gain de cohésion au moment de la rupture de l'échantillon. Le tableau IX donne les valeurs de résistance à la traction en service des nappes plastiques en feuilles.

\section{2}

\section{Renforcement par des nappes discontinues}

\section{and \\ Expérimentation}

L'étude des nappes discontinues est conduite comparativement avec celle des nappes continues, en faisant varier deux paramètres : la dimension des élé-

TABLEAU VIII Valeurs du gain en cohésion théorique et expérimentale apporté par la présence des nappes continues.

Experimental and theoretical values of the cohesion gain due to continuous layers.

\begin{tabular}{l|r|r|r|r|r} 
Types d'essais & 1 & 2 & 3 & 4 & 5 \\
\hline$\Delta C_{e p p}(k P a)$ & 0 & 5 & 9 & 15 & 22 \\
\hline$\Delta C_{\mathrm{in}}=f(\phi)(\mathrm{kPa})$ & 0 & 13 & 13 & 25 & 25 \\
\hline
\end{tabular}

TABIEAUIX Valeurs de résistance à la traction en service des nappes plastiques en feuilles.

\begin{tabular}{|c|c|c|}
\hline Types d'essais & $\begin{array}{l}\Delta C_{\text {oxp }} \\
(\mathrm{KPa})\end{array}$ & $\begin{array}{c}\mathrm{T}_{\mathrm{p}}=\Delta \mathrm{C} \quad \mathrm{S} / \mathrm{b} \cdot \mathrm{tg} \phi \\
(\mathrm{kN} / \mathrm{m})\end{array}$ \\
\hline 1 & 0 & 0 \\
\hline 2 & 5 & 1,6 \\
\hline 3 & 9 & 2,8 \\
\hline 4 & 15 & 4,7 \\
\hline 5 & 22 & 6,9 \\
\hline
\end{tabular}
Values of the tensile strength for plastic layers in sheets. 
TABLEAUX Valeurs de résistances à la traction en service des nappes plastiques en morceaux. Values of the tensile strength for plastic layers in pieces.

\begin{tabular}{|c|c|c|c|c|c|c|c|c|c|}
\hline & Masse $(g)$ & 60 & 120 & 210 & 270 & 300 & 540 & 660 & 1200 \\
\hline & Masse surfacique $M_{x}\left(\mathrm{~kg} / \mathrm{m}^{\dagger}\right)$ & 0,25 & 0,5 & 0,87 & 1,1 & 1,2 & 2,2 & 2,7 & 5 \\
\hline $\begin{array}{l}\text { De morceaux plastiques } \\
(60 \mathrm{~cm} \times 40 \mathrm{~cm})\end{array}$ & $\begin{array}{l}\Delta \mathrm{C}_{\text {exp }}(\mathrm{kPa}) \\
\mathrm{T}_{\mathrm{p}}=\Delta \mathrm{C}_{\mathrm{em}} \mathrm{S}_{\mathrm{c}} / \mathrm{L} \cdot \mathrm{tg} \phi \\
(\mathrm{kN} / \mathrm{m})\end{array}$ & $\begin{array}{l}5,1 \\
1,6\end{array}$ & $\begin{array}{r}14,8 \\
5,2\end{array}$ & 6,3 & & & & & \\
\hline $\begin{array}{l}\text { Grave renforcée par nappe } \\
\text { de morceaux plastiques } \\
(30 \mathrm{~cm} \times 40 \mathrm{~cm} \text { ) }\end{array}$ & $\begin{array}{l}\Delta C_{\operatorname{exg}}(\mathrm{kPa}) \\
\mathrm{T}_{\mathrm{P}}=\Delta \mathrm{C}_{\operatorname{ma}} \mathrm{S} / \mathrm{L} \cdot \mathrm{tg} \phi \\
(\mathrm{kN} / \mathrm{m})\end{array}$ & & & & $\begin{array}{l}7 \\
2,2\end{array}$ & $\begin{array}{l}8 \\
2.5\end{array}$ & $\begin{array}{r}13,3 \\
4,2\end{array}$ & $\begin{array}{r}15,6 \\
4,9\end{array}$ & $\begin{array}{r}18,7 \\
5,9\end{array}$ \\
\hline $\begin{array}{l}\text { Grave renforcée par nappe } \\
\text { de morceaux plastiques } \\
(20 \mathrm{~cm} \times 40 \mathrm{~cm})\end{array}$ & $\begin{array}{l}\Delta C_{\text {exe }}(\mathrm{kPa}) \\
T_{\mathrm{g}}=\Delta \mathrm{C}_{\text {exp }} \mathrm{S} / \mathrm{L} \cdot \mathrm{tg} \phi \\
(\mathrm{kN} / \mathrm{m})\end{array}$ & & & & $\begin{array}{l}5 \\
1,6\end{array}$ & $\begin{array}{l}6 \\
1,9\end{array}$ & $\begin{array}{r}11,4 \\
3,6\end{array}$ & $\begin{array}{r}13,6 \\
4,3\end{array}$ & $\begin{array}{r}16,2 \\
5,1\end{array}$ \\
\hline $\begin{array}{l}\text { Grave renforcée par nappe } \\
\text { de morceaux plastiques } \\
(10 \mathrm{~cm} \times 40 \mathrm{~cm})\end{array}$ & $\begin{array}{l}\Delta \mathrm{C}_{\text {opg }}(\mathrm{kPa}) \\
\mathrm{T}_{\mathrm{B}}=\Delta \mathrm{C}_{\text {exp }} \mathrm{S} / \mathrm{L} \text {.tg } \phi \\
0 \mathrm{kN} / \mathrm{m})\end{array}$ & & & & $\begin{array}{l}2,8 \\
0,9\end{array}$ & $\begin{array}{l}3,1 \\
1\end{array}$ & $\begin{array}{l}5,7 \\
1.8\end{array}$ & $\begin{array}{l}6.6 \\
2.1\end{array}$ & $\begin{array}{l}7,6 \\
2,4\end{array}$ \\
\hline $\begin{array}{l}\text { Grave renforcée par nappes } \\
\text { en déchets plastiques }\end{array}$ & 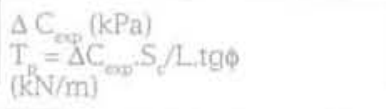 & & & & $\begin{array}{l}5,7 \\
1,8\end{array}$ & $\begin{array}{l}6,6 \\
2,1\end{array}$ & $\begin{array}{c}12,7 \\
4\end{array}$ & $\begin{array}{r}14,6 \\
4,6\end{array}$ & $\begin{array}{r}17,1 \\
5,4\end{array}$ \\
\hline
\end{tabular}

ments et leur répartition en masse. L'objectif de ces essais est de trouver une relation entre l'apport en cohésion des nappes discontinues et l'apport des nappes continues afin de pouvoir caracteriser les nappes discontinues par une certaine résistance à la traction $T_{p}$ qui est calculée à partir de la formule 13 :

$$
T_{p}=\Delta C_{e x p} \cdot S / \text { L.tgф }
$$

Contrairement à l'approche par nappe continue, cette résistance à la traction $T_{\text {n }}$ ne correspond pas à la rupture d'un élément mais à la rupture de la nappe par glissement des éléments les uns par rapport aux autres. Le tableau $\mathrm{X}$ donne l'ensemble des résultats où l'on constate évidemment la croissance de $T_{p}$ en fonction de l'augmentation de la taille des éléments.

L'évolution du rapport de la contrainte de cisaillement maximale (R.C.M. $=\tau / \tau$ ) en fonction de la forme géométrique des morceaux plastiques est représentée sur la figure 19 ( $\tau$ et $\tau_{0}$ sont des contraintes de cisaillement du gravier renforcé et non renforcé). Pour une taille donnée, $T$ croît avec l'augmentation de la masse jusqu'à une valeur palier (Fig. 20). Les essais de graves renforcées par nappes discontinues de longueur de

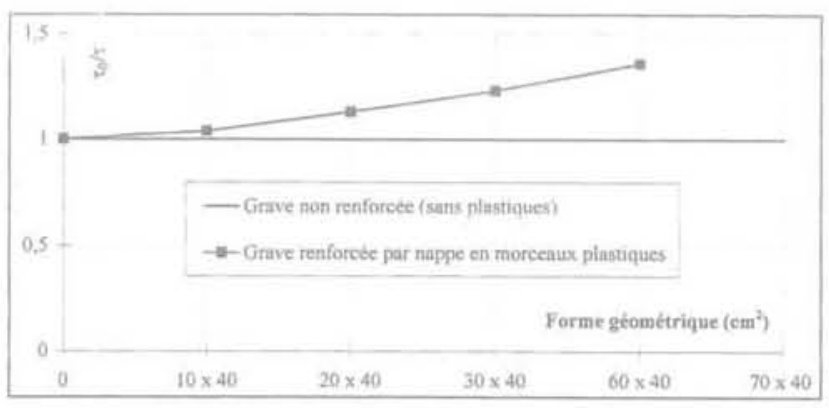

FG.19 Évolution du rapport de la contrainte de cisaillement maximale ( $\mathrm{RCM}$ ) en fonction de la forme géométrique des morceaux plastiques $\left(\sigma=54,5 \mathrm{kPa}\right.$ et $\left.\mathrm{M}_{\mathrm{s}}=1 \mathrm{~kg} / \mathrm{m}^{2}\right)$. Evolution of the maximum shear tensile strength ratio according to the geometrical form of plastic pieces $\left(\sigma=54,5 \mathrm{kPa}\right.$ and $\mathrm{M}_{\mathrm{a}}=$ $\left.1 \mathrm{~kg} / \mathrm{m}^{2}\right)$. recouvrement de moins de $10 \mathrm{~cm}$ ont été exclus car nous estimons que l'apport du renforcement dans ce cas est négligeable. Pour mieux approcher la réalité des renforts par déchets, des essais ont été également effectués avec des nappes formées de sacs d'emballage souples.

\section{2.}

\section{Exploitation}

La courbe 20 permet de faire la correspondance entre la résistance à la traction des nappes discontinues et celle équivalente des nappes continues. Le rapport d'équivalence $R_{\mathrm{r}}$ varie fortement avec d'une part la taille des éléments de renfort et d'autre part la résistance à la traction recherchée (tableau XI).

On peut noter que pour obtenir une continuité équivalente satisfaisante, la taille des éléments doit être au minimum de $30 \mathrm{~cm}$. Lorsqu'elle atteint $60 \mathrm{~cm}$, la valeur de $R_{E}$ est égale à 6 pour des résistances à la traction $T_{\text {p }}$ de $8 \mathrm{kN} / \mathrm{m}$. Dans ce cas, on obtient des résultats tout a fait comparables avec certains géotextiles.

\section{6}

\section{Conclusion}

L'étude comparative des deux boîtes (à plan de cisaillement horizontal et vertical) a abouti aux remarques suivantes:

- le pic de la contrainte tangentielle est très prononcé dans le cas de la boite à plan de cisaillement ver. tical, ceci est dủ au phénomène de dilatance qui est important dans cette boîte ;

- la valeur de l'angle de frottement moyen gravegrave déterminée par l'essai à plan de cisaillement horizontal est du même ordre que la valeur de l'angle de frottement interne déterminée dans un essai à plan de cisaillement vertical ;

- on note une certaine analogie qualitative de la variation du coefficient de frottement apparent en fonction de la contrainte normale pour les deux types d'essai ; 


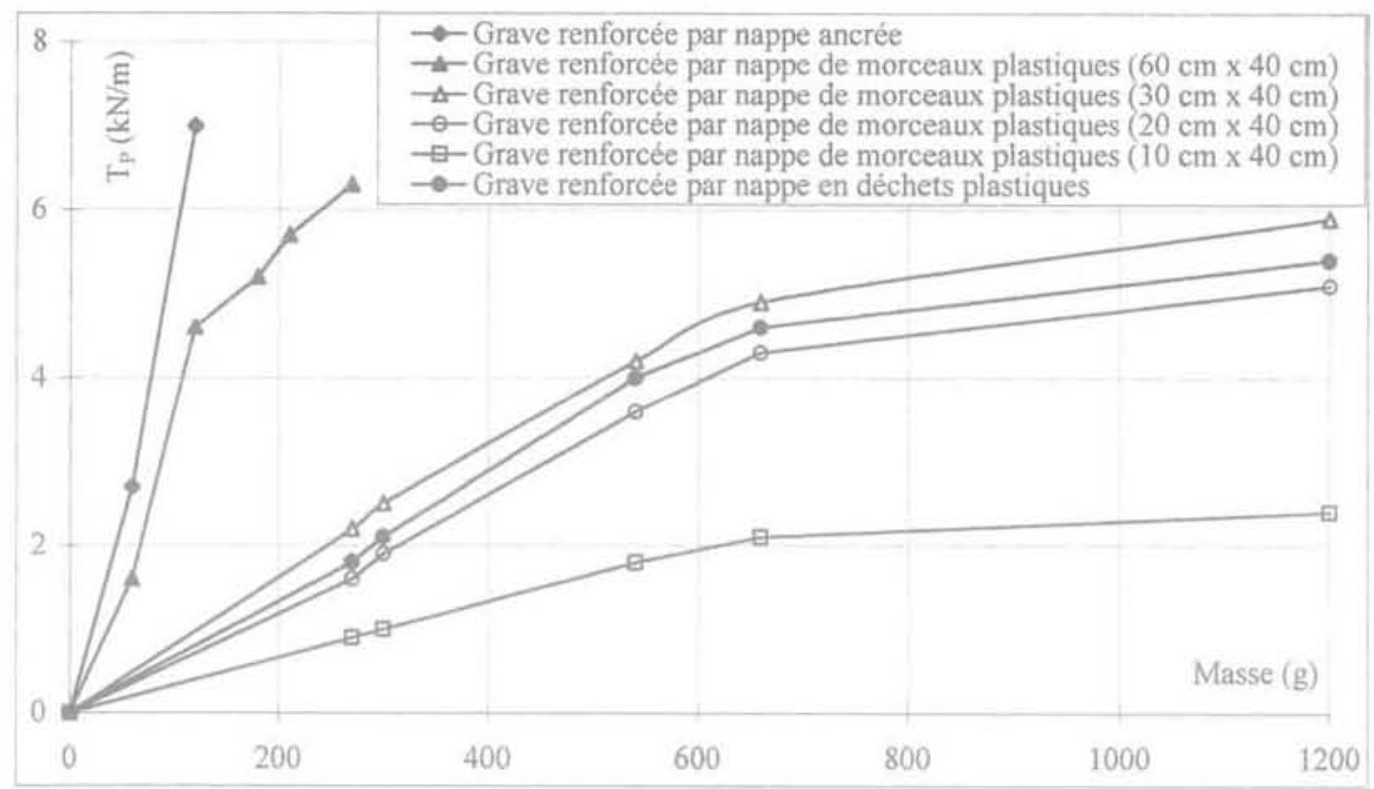

FG. 20 Résistance à la traction en service de la nappe en matières plastiques. The tensile strength of the layer of plastic materials.

TABLEAUXI Valeurs des rapports équivalents des quantités de matières plastiques pour une résistance en service donnée.

Values of the tensile strength for plastic layers in pieces.

\begin{tabular}{c|c|c|c|c|c}
\multirow{2}{*}{$\begin{array}{c}T \\
(\mathrm{KN} / \mathrm{m})\end{array}$} & $\begin{array}{c}\text { Grave renforcée } \\
\text { par nappe de MP } \\
\left(60 \times 40 \mathrm{~cm}^{-1}\right)\end{array}$ & $\begin{array}{c}\text { Grave renforcée } \\
\text { par nappe de MP } \\
\left(30 \times 40 \mathrm{~cm}^{-1}\right)\end{array}$ & $\begin{array}{c}\text { Grave renforcée } \\
\text { par nappe de MP } \\
\left(20 \times 40 \mathrm{~cm}^{-1}\right)\end{array}$ & $\begin{array}{c}\text { Grave renforcée } \\
\text { par nappe de MP } \\
\left(10 \times 40 \mathrm{~cm}^{2}\right)\end{array}$ & $\begin{array}{c}\text { Grave renforcée } \\
\text { par nappe en } \\
\text { déchets plastiques }\end{array}$ \\
\hline 2 & 2 & 6,8 & 8,1 & 16 & 7,8 \\
\hline 3 & 1,6 & 6,6 & 7,9 & 6,9 \\
\hline 4 & 1,7 & 7,1 & 7,8 & 7.5 \\
\hline 5 & 1,8 & 7,6 & 9,7 & & 7,8 \\
\hline
\end{tabular}

$R_{\varepsilon}{ }^{*}=$ (Masse des monceaux des nappes discontinues)/(Masse des feuilles des nappes continues).

- liessai à plan de cisaillement vertical est préféré pour l'étude du comportement de cisaillement du sol, vu sa représentativité de la réalité lors d’un glissement de terrain.

Les essais effectués avec des nappes plasticques non ancrées mettent en évidence une résistance moins grande que dans le cas des nappes plastiques ancrées, surtout pour un déplacement important. Cette différence est la conséquence d'une efficacité considérable de résistance à la traction de la part des feuilles plastiques lorsque celles-ci sont ancrées.

Le plastique augmente nettement la résistance au cisaillement lorsqu'il est mis en tension sous des grands déplacements.

L'adjonction aux sols de nappes d'éléments de matières plastiques discontinus entraine une amélioration notable des caractéristiques mécaniques des sols. Cette amélioration a été quantifiée avec le calcul d'une pseudo-cohésion.

Il apparaît que l'effet maximal sur la résistance de cisaillement est obtenu avec les nappes continues. Mais plus la masse et la taille des matières plastiques augmentent, plus le chevauchement et le recouvrement entre les morceaux sont importants. Le comportement du renfort discontinu tend vers celui de la nappe continue.

L'utilisation de déchets plastiques souples est donc possible pour renforcer des sols de remblais. Cette technique, reprise par le procédé Plasterre, permet de valoriser ces déchets et de résoudre le problème posé par leur élimination. 


\section{Bibliographie}

Boulon M., Hoteit N. - $\approx$ Interface solinclusion. Comportement de l'essai de cisaillement direct à rigidité normale imposée D. Rapport GRECO, 1988. p. 314-321.

Coulet C., Rakotondramanitra J.D. Bacot J. - "Soil reinforcement making use of waste plastic materials study with large shear box machine w. VIIIth Nat. Conf on Soil Mechanics and Foundation Engineering, Wroclaw, Pologne, 1987. Coulet C., Rakotondramanitra J.D., Bacot J.
- « Utilisation des déchets de matières plastiques pour le renforcement des

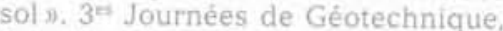
Saint-Nazaire 1987

Lareal P. Bourdeau Y. $-\alpha$ Essais de cisaillement in situ sur des sols alluvionnaires dans la vaillée du Rhône $x$. Travaux $n^{*} 459-460$, juin-juillet 1973, D $52-59$

Maes M. - Option déchets, Paris, Johanet 1993, $621 \mathrm{p}$.

Messa T. Azzouz R., Coulet C., Curtil L. "Soil reinforcement by using layers of soft or discontinuous plastic wastes 11 . GREEN2 - 2nd International Symposium on Geotechnics and the Environment, 8-11 September 1997, Krakow, Poland.

Messas T, - « Étude des renforcements des sols par nappes discontinues en matières plastiques souples $n$. Thèse présentée à l'universilé Claude-Bernard de Lyon (UCBL), soutenue le 6 novembre 1997, pour obtenir le grade de docteur. 